\title{
Infrasound detection and altitude estimation associated with the December 22, 2020 Yushu fireball
}

Wei Cheng ${ }^{1,2}$, Pengxiao Teng ${ }^{1 *}$, Jun Lyu ${ }^{1}$ and Yijing Dai ${ }^{1,2}$

\begin{abstract}
A large bolide was reported at 23:23:33 UTC on December 22, 2020, at a height of $\sim 35.5 \mathrm{~km}$ at $31.9^{\circ} \mathrm{N}, 96.2^{\circ} \mathrm{E}$ in Yushu, Qinghai Province, China. It is the largest fireball observed in China on record with a TNT equivalent of 9.5 kilotons. Infrasound signals were detected by a four-element infrasound array deployed in Yunnan Province, China. The parameters of this event were obtained using the progressive multi-channel correlation method. The altitude of this event was estimated to be $43.22 \pm 15.51 \mathrm{~km}$ using a ray tracing back-projection algorithm.
\end{abstract}

Keywords: Infrasound, Fireball, Altitude, Ray tracing back-projection algorithm

\section{Introduction}

The seismic arrays in Qinghai Province (China) detected the fall of a meteor on December 22, 2020 (Meteorite is found out 2020). According to public information from the Jet Propulsion Laboratory (JPL), the estimated energy of this fireball is 9.5 kilotons (kt) TNT equivalent, which makes this the largest meteoritic event ever recorded in China. Infrasound signals can be emitted by a meteor explosion, and they have been used to investigate the acoustic wave parameters and blast energy of meteor impacts in previous studies (Le Pichon et al. 2013; Caudron et al. 2016).

Fireballs can be recorded by seismic stations in the area close to the impact site, and the size of a meteor can be estimated based on the fundamental frequency (Corentin et al. 2016). However, if the event occurs over the vast ocean and the impact energy is not large enough, the bolide parameter can only be evaluated from satellite data (Silber et al. 2009). In 2013, the infrasound data generated by an enormous energy burst from a small asteroid

\footnotetext{
${ }^{*}$ Correspondence: px.teng@mail.ioa.ac.cn

${ }^{1}$ Key Laboratory of Noise and Vibration Research, Institute of Acoustics,

Chinese Academy of Science, Beijing 100190, China

Full list of author information is available at the end of the article
}

near the city of Chelyabinsk, Russia, was recorded by arrays in the USA at distances of $6000 \mathrm{~km}$ to $10,000 \mathrm{~km}$ from the source (Hedlin et al. 2014). Infrasound observations can be used to estimate parameters, such as the azimuth and energy. The equivalent TNT size of a fireball explosion can be estimated from the period of the signal received for bolides, which are sufficiently large (ReVelle 1997).

In this study, the infrasound recordings associated with the December 2020 Yushu fireball were investigated using the progressive multi-channel correlation (PMCC) method and the altitude of this event estimated using the ray tracing back-projection algorithm (Cansi 1995; Shang et al. 2019).

\section{Method and results}

The Yushu fireball occurred at 23:23:33 UTC on December 22, 2020, over Qinghai Province, China. The coordinates of this event were about $31.9^{\circ} \mathrm{N}, 96.2^{\circ} \mathrm{E}$, and the meteor shone brightly across the sky. It is suspected to have landed near Yushu County according to China's Earthquake Network Centre (Qinghai Fireball IncidentInvestigation 2020). The distance between the infrasound array in Yunnan Province which was deployed to forewarn earthquake and this event was about $1100 \mathrm{~km}$ 
(Fig. 1). The array $\left(25.9^{\circ} \mathrm{N}, 102.3^{\circ} \mathrm{E}\right)$ consists of four $\mathrm{CDC}-2 \mathrm{~B}$ infrasound sensors which ranges from 0.02 to $20 \mathrm{~Hz}$ in frequency band. The sample rate of data acquisition unit (DAU) is $100 \mathrm{~Hz}$. On December 23, 2020, the array detected an infrasound event at 00:14:03 UTC. The PMCC method was used to estimate the wave parameters using 15 logarithmically spaced frequency bands ranging from 0.02 to $50 \mathrm{~Hz}$ with second order Chebyshev filters which is more stable than third and fourth order Chebyshev filters here (Garces 2013). The time-window length maintained a constant period of $50 \mathrm{~s}$ and was time-shifted by $25 \%$ of the window length.

As shown in Fig. 2, two arrivals were detected at 00:14:03-00:17:52 and 00:18:44-00:28:57, which are marked as A and B, respectively. The back-azimuth of arrival $\mathrm{A}$ is $337^{\circ}$ and that of arrival $\mathrm{B}$ is $326^{\circ}$, which correspond to the position reported by JPL. In the lower panel of Fig. 2, there seems to be an event at about 00:35:00. The signal at 00:35:00 has no corresponding PMCC family detected. This may be an interfering sound source in the array geometry, because the amplitude of signal is obviously different.

In addition to the Yunnan infrasound array, the Xinjiang infrasound array $\left(40.7^{\circ} \mathrm{N}, 84.4^{\circ} \mathrm{E}\right)$ deployed for forewarning earthquake is located $1300 \mathrm{~km}$ from the fireball event. However, this array failed to observe the signal from the fireball with PMCC method (Fig. 3). Simulation of the infrasound in the atmosphere was utilized to explain this. To demonstrate the propagation traces of the infrasound signal, the nonlinear progressive wave equation (NPE) was used to simulate the variation in the transmission loss of the infrasound signal with distance (McDonald et al. 2011). The influence of atmospheric winds was approximated by adding the horizontal wind in the direction from the source toward the receiver and the sound speed in the stratified atmosphere. The result labeled as the effective sound speed was used to simulate the infrasound propagation in an atmosphere with sufficiently low wind speeds. Models Mass Spectrometer and Incoherent Scatter Radar Extended from the Ground through Exosphere (MSISE00) provides temperature profile which can be utilized to calculate the sound speed profile (Picone et al. 2002). Horizontal Wind Model (HWM14) can provide horizontal wind profile from the ground to an altitude of $150 \mathrm{~km}$ (Hedin et al. 1998). The effective sound speed profile can be obtained by adding them.

The effective sound speed profiles in the direction toward the infrasound array in Yunnan Province and Xinjiang Province are shown in Fig. 4. The maximum value of the profile between altitude $30-60 \mathrm{~km}$ in the direction toward Yunnan Station is bigger than that on the ground, which means the waveguide between stratosphere and the ground can be formed (Yang et al. 2007). The situation in the direction toward Xinjiang Station is different. The maximum value of effective sound speed in altitude $30-60 \mathrm{~km}$ is smaller than that on the ground. This means the energy propagated through the waveguide between stratosphere and the ground leaked into the area between stratosphere and thermosphere.

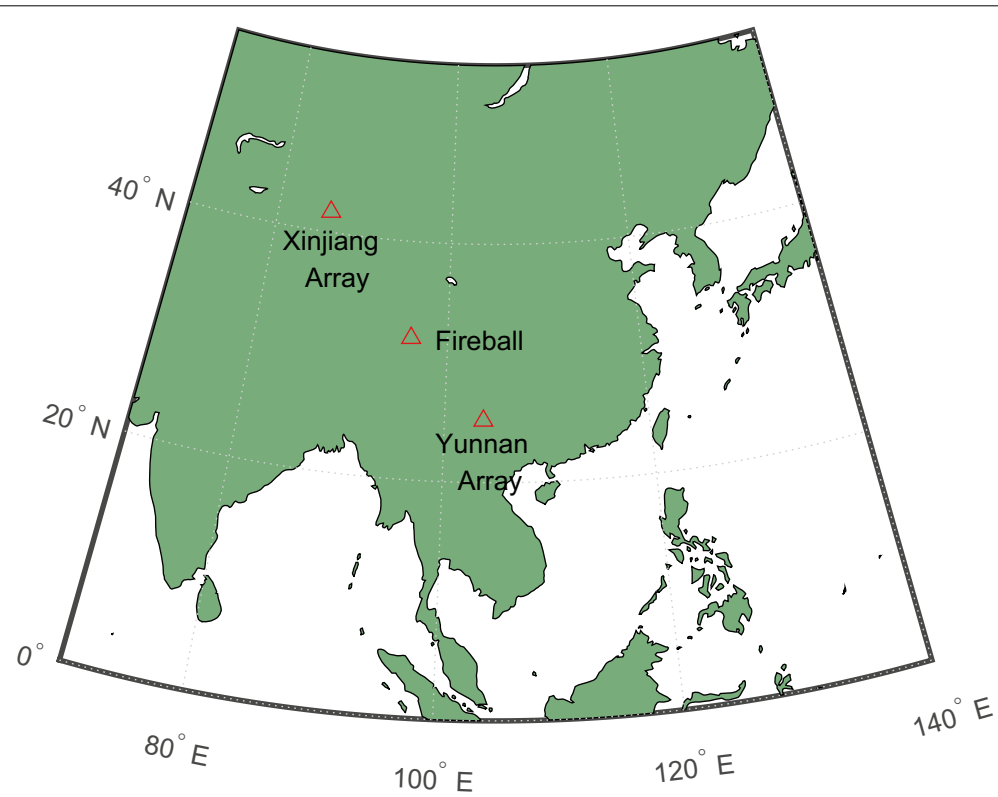

Fig. 1 Map. Locations of the infrasound station in Yunnan Province and the fireball event 

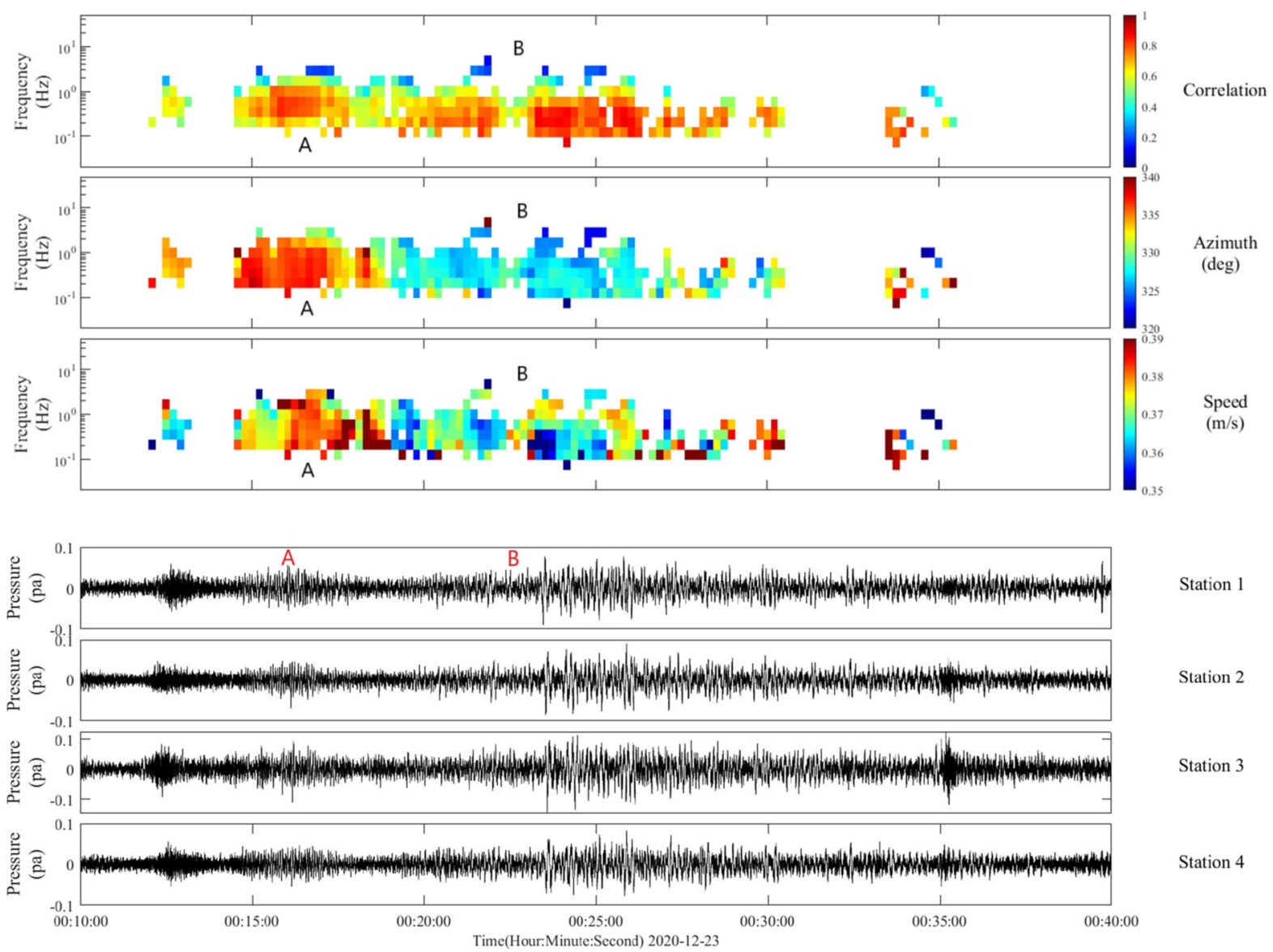

Station 1

Station 2

Station 3

Station 4

Fig. 2 PMCC results for the data from the infrasound station in Yunnan Province, China. According to the corresponding relationship between the PMCC family and the amplitude of the signal, the received signal can be divided into arrivals A and B, which are marked above. The azimuths and elevation angles (apparent velocity) of arrivals $A$ and $B$ are significantly different. The main frequency band of arrival B is lower than that of arrival $A$ according to the correlation
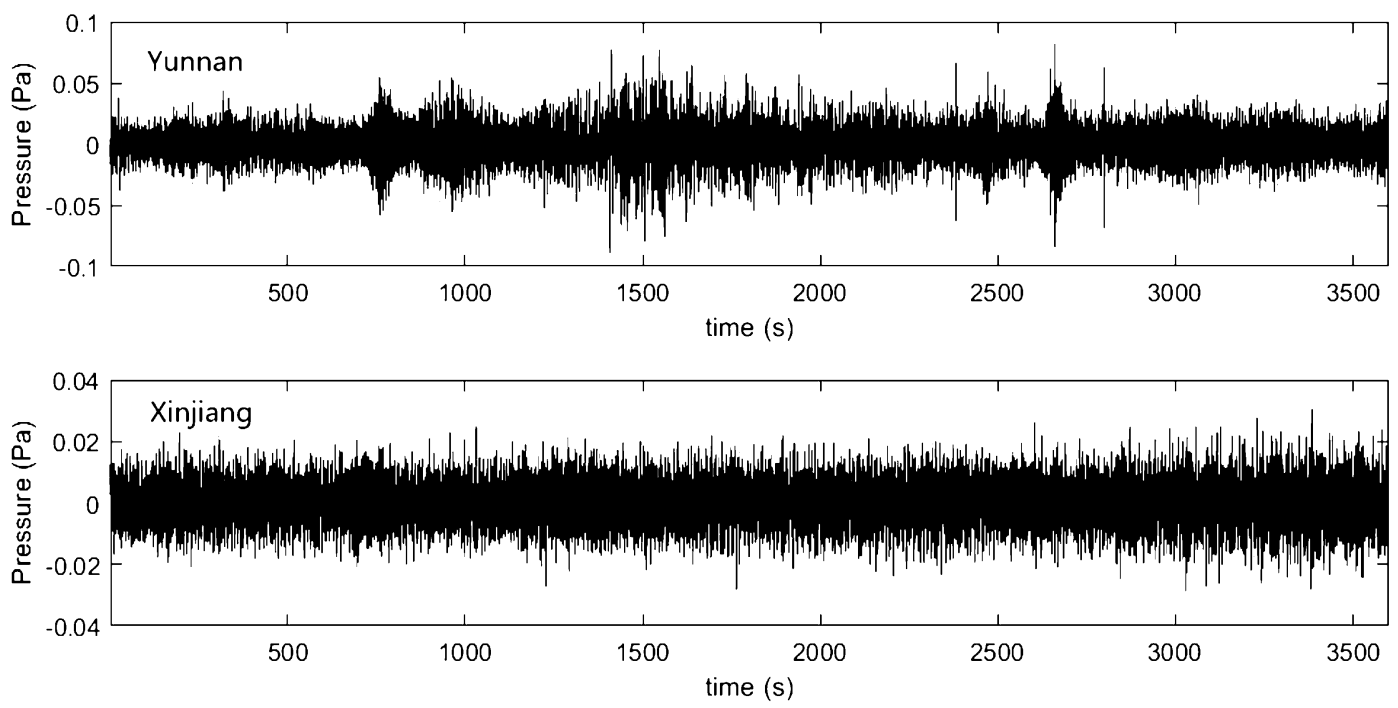

Fig. 3 Signals received by the Yunnan and Xinjiang infrasound arrays 


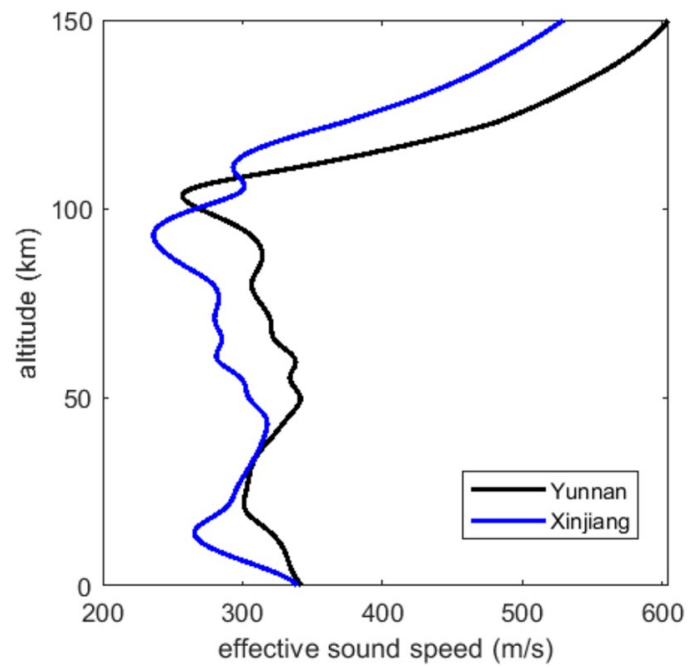

Fig. 4 Effective sound speed profile in the direction toward Yunnan Station (black line) and Xinjiang Station (blue line)

As shown in Fig. 5a, the most of infrasound energy is reflected to ground, which facilitates to propagate far in this waveguide between stratosphere and the ground. In contrast, a large part of infrasound energy propagated upward into thermosphere in Fig. 5b, which causes the large attenuation of the signal. The attenuation is mainly caused by the classical absorption and relaxation absorption (Sutherland et al. 2004).

\section{Discussion}

The altitude of the infrasound event is not a trivial factor in the estimation because of the influence that the inhomogeneous atmosphere has on the infrasound propagation trace. The height of the infrasound source has a significant effect on the recognition of the type of source. The ray tracing back-projection algorithm can be used to estimate the height of this event. The speed of sound $c$ at the center of the array was assumed to be $0.337 \mathrm{~km} / \mathrm{s}$. The elevation angle of arrival A from the horizontal can be calculated from the apparent velocity $c_{a p}$ using $\arccos \left(\mathrm{c} / \mathrm{c}_{\mathrm{ap}}\right)$. Considering the typical height of the fireball was initially observed and reported by JPL, we chose the first family in the results of the PMCC, which corresponds to arrival A. The elevation
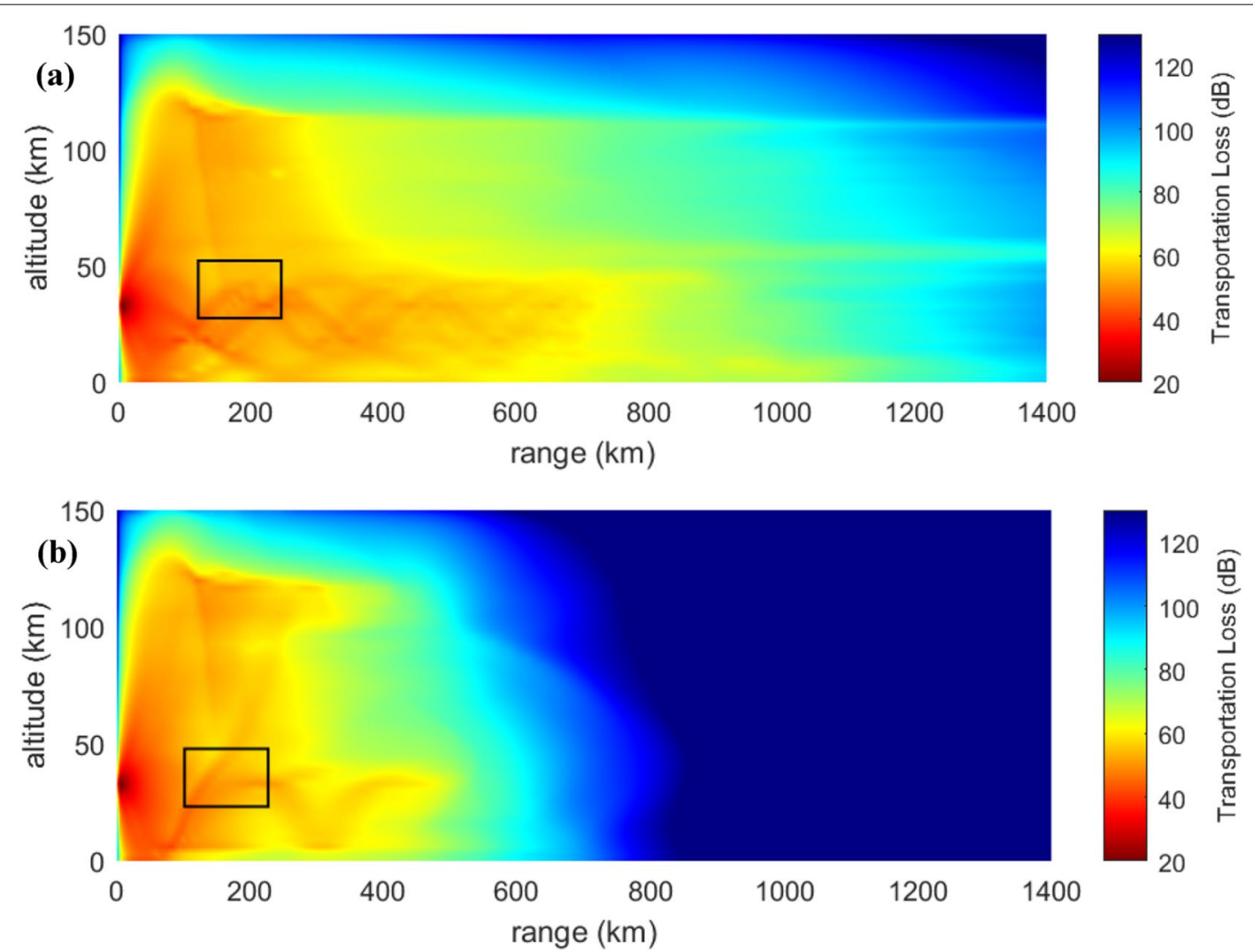

Fig. 5 a Distribution of the transportation loss of the infrasound signal in the direction of Yunnan Station obtained using the NPE method. The transportation of the Yunnan array is $74 \mathrm{~dB}$; and $\mathbf{b}$ distribution of the transportation loss of the infrasound signal in the direction of Xinjiang Station obtained using the NPE method. The transportation loss of the Xinjiang array is $175 \mathrm{~dB}$ 


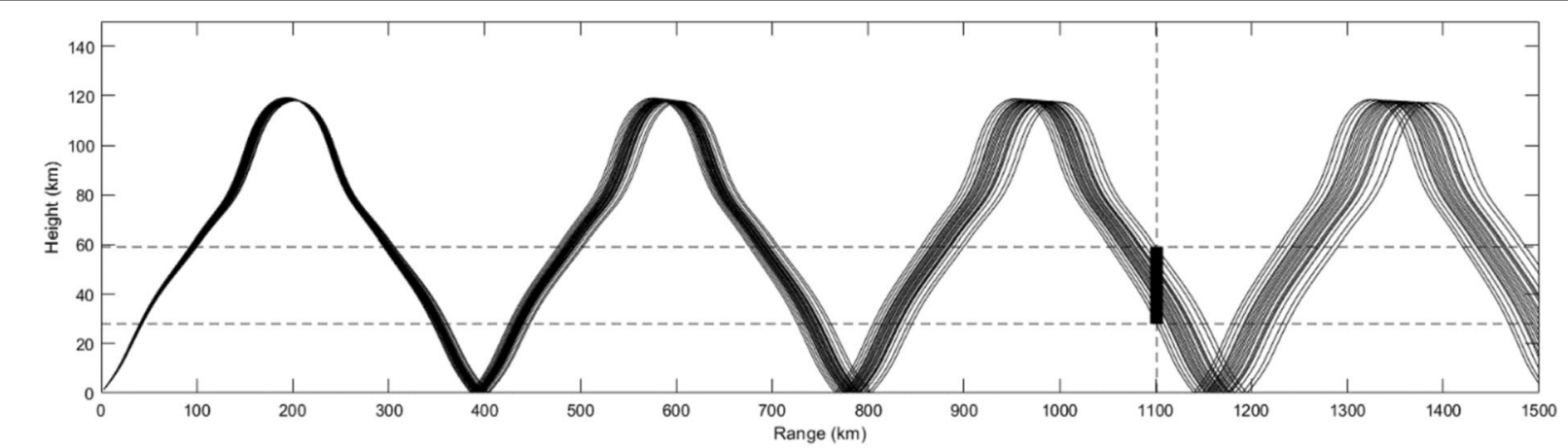

Fig. 6 Back-projection reconstruction of the ray trajectories of the signal at 00:14:23 recorded at the infrasound station in Yunnan Province

angle of this family is $24^{\circ}-26.8^{\circ}$, and the distance between the source and the array is $1100 \mathrm{~km}$.

As shown in Fig. 6, the altitude at a distance of $1100 \mathrm{~km}$ ranged from 27.7 to $58.7 \mathrm{~km}$, with an average altitude of $43.22 \mathrm{~km}$, while the height reported by JPL is $35.5 \mathrm{~km}$. The error relative to the reported altitude, which was obtained from satellites, is $21.75 \%$. The discrepancy is caused by the ambiguity of the apparent velocity, and the observed height is within the estimated domain.
The released energy of the fireball can be estimated using the period at the maximum amplitude (ReVelle 1997):

$$
\log _{10}(E / 2)=3.34 \log _{10}(T)-2.58 \quad \frac{E}{2} \leq 100 k t,
$$

where $E$ is the energy in kilotons; and $T$ is the period in seconds. As shown in Fig. 2, the signal received in Yunnan infrasound array can been divided into arrival A and B, which are, respectively, corresponding to two families in PMCC result. The signal around the maximum amplitude
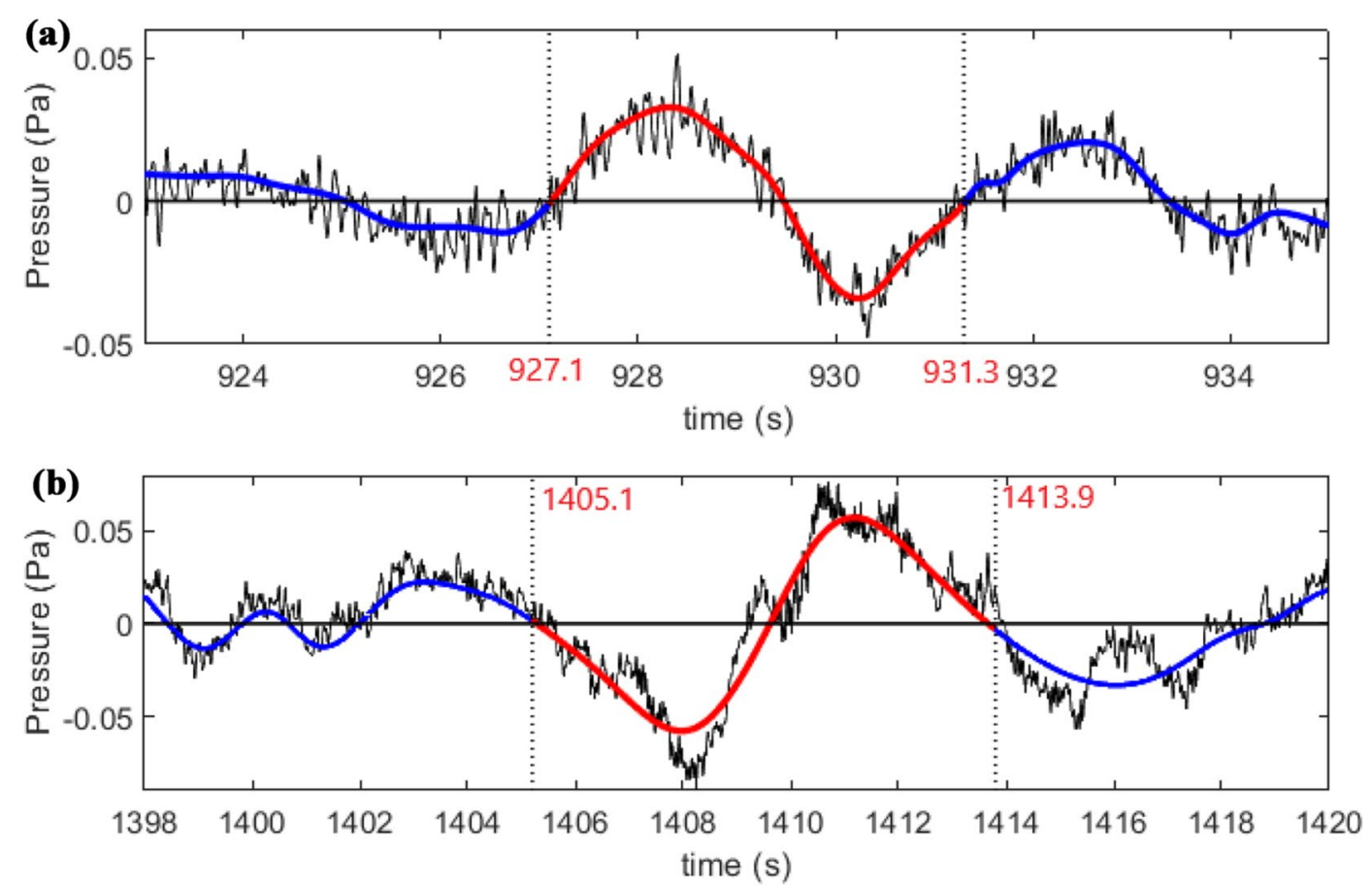

Fig. 7 Black curve is the original signal, the blue bold curve is the outline of signal from EMD procedure, the vertical straight line is the zero-pressure reference line, the vertical dotted line is starting time and ending time. $\mathbf{a}$ Period at the maximum amplitude of arrival $\mathrm{A}$ is $4.2 \mathrm{~s}$; and $\mathbf{b}$ period at the maximum amplitude of arrival $\mathrm{B}$ is $8.8 \mathrm{~s}$ 
of arrival A and B is shown in Fig. 7. To exclude the detail and estimate the period, empirical mode decomposition (EMD) is utilized to obtain the dominant period of signal (Huang et al. 1998). As shown in Fig. 7, the period of original signal can be estimated using that of outline. The period at the maximum amplitude of arrival $\mathrm{A}$ is $4.2 \mathrm{~s}$ and that of arrival B is $8.8 \mathrm{~s}$. The estimated energies obtained using this method based on the infrasound signal is 0.64 $\mathrm{kt}$ for arrival A and $7.51 \mathrm{kt}$ for arrival B. Using the optical energy measured by the space-based sensors, the equivalent TNT size of the fireball can be estimated for comparison (Brown et al. 2002). The energy of arrival A is much lower than the typical equivalent of $7.51 \mathrm{kt}$, and that of arrival $B$ is much closer to this value. This indicates that arrival $\mathrm{B}$ corresponds to the fireball event recorded by the optical sensors.

\section{Conclusions}

A large bolide was observed at dawn over Qinghai Province near the city of Yushu, and two arrivals were received. An infrasound array deployed in Yunnan Province, which is $1100 \mathrm{~km}$ away from the event, detected the arrival using four $\mathrm{CDC}-2 \mathrm{~B}$ infrasound sensors. To estimate the altitude of the source of the first arrival, the ray tracing back-projection algorithm was used to obtain a result of $43.22 \pm 15.51 \mathrm{~km}$, which is approximately the reported altitude of $35.5 \mathrm{~km}$. Considering that only one array detected the infrasound signal, it is expected that the estimated altitude will be more reliable with more data from arrays in different directions and ranges. The longest period of each arrival was used to estimate the energy of this event, and the result $7.51 \mathrm{kt}$ for arrival B indicates that this arrival corresponds to the fireball observed by the optical sensors.

\section{Authors' contributions \\ CW analyzed data and wrote the manuscript; TP contributed to analysis and manuscript preparation; LJ helped perform the analysis with constructive discussions; DY helped analyze data. All authors read and approved the final manuscript.}

\section{Funding}

Project supported by the National Natural Science Foundation of China (Grant Nos.11774372, 11874389), The Institute of Acoustics of the Chinese Academy of Science Youth Talents Program (Grant No. QNYC201811).

\section{Availability of data and materials}

Not applicable.

\section{Declarations}

\section{Competing interests}

The authors declare that they have no competing interests.

\section{Author details}

${ }^{1}$ Key Laboratory of Noise and Vibration Research, Institute of Acoustics, Chinese Academy of Science, Beijing 100190, China. ${ }^{2}$ University of Chinese Academy of Science, Beijing 101408, China.

Received: 25 January 2021 Accepted: 7 July 2021

Published online: 23 July 2021

\section{References}

'A meteorite is found out to fall in Yushu, Qinghai. http://www.chinanews. com/sh/2020/12-24/9369907.shtml. Accessed 24 Dec 2020

Brown P, Spalding RE, ReVelle DO, Tagliaferri E, Worden SP (2002) The flux of small near-earth objects colliding with the earth. Nature 420(6913):294-296

Cansi Y (1995) An automatic seismic event processing for detection and location: the PMCC method. Geophys Res Lett 22(9):1021-1024

Caudron C, Taisne B, Perttu A, Garcés M (2016) Infrasound and seismic detections associated with the 7 september 2015 bangkok fireball. Geosci Lett 3(1):26

De Groot-Hedlin CD, Hedlin MAH (2014) Infrasound detection of the Chelyabinsk meteor at the USarray. Earth Planet Sci Lett 402:337-345

Edward McDonald B, Piacsek AA (2011) Nonlinear progressive wave equation for stratified atmospheres. J Acoust Soc Am 130(5):2648

Garces MA (2013) On infrasound standards, part1 time, frequency, and energy scaling. Inframatics 02(2):13-35

Hedin AE, Spencer NW, Killeen TL (1998) Empirical global model of upper thermosphere winds based on atmosphere and dynamics explorer satellite data. J Geophys Res 93:9959-9978

Huang NE, Shen Z, Long SR, Wu SC, Shih HH, Zheng Q, Yen NC, Tung CC, Liu $\mathrm{HH}$ (1998) The empirical mode decomposition and the Hilbert spectrum for nonlinear and non-stationary time series analysis. Proc R Soc Lond A Math 454:903-995

Le Pichon A, Ceranna L, Pilger C, Mialle P, Brown D, Herry P, Brachet N (2013) The 2013 Russian fireball largest ever detected by CTBTO infrasound sensors. Geophys Res Lett 40(14):3732-3737

Picone JM, Hedin AE, Drob DP, Aikin AC (2002) NRLMSISE-00 empirical model of the atmosphere: Statistical comparisons and scientific issues. J Geophys Res Space Phys 107(A12):1468

'Qinghai Fireball Incident-Investigation of the Identity of the "Perpetrator". https://www.sohu.com/a/441232060_610722. Accessed 29 Dec 2020

ReVelle DO (1997) Historical detection of atmospheric impacts by large bolides using acoustic-gravity waves. Annals of the New York Academy of Sciences

Shang C, Teng P, Lyu J, Yang J, Sun H (2019) Infrasonic source altitude localization based on an infrasound ray tracing propagation model. J Acoust Soc Am 145(6):3805-3816

Silber EA, ReVelle DO, Brown PG, Edwards WN (2009) An estimate of the terrestrial influx of large meteoroids from infrasonic measurements. J Geophys Res 114:E8

Sutherland LC, Bass HE (2004) Atmospheric absorption in the atmosphere up to $160 \mathrm{~km}$. Acoust Soc Am J 115(3):1012-1032

Yang X, Chen Y (2007) Atmospheric acoustic. 55-59

\section{Publisher's Note}

Springer Nature remains neutral with regard to jurisdictional claims in published maps and institutional affiliations. 